\title{
Three case reports of simple vascular malformations: inappropriate, perplexing and interchangeable use of nosology by clinicians and pathologists
}

\begin{abstract}
Scientific articles and newer editions of medical text books show significant misapprehension among authors and scientific fraternities over the correct nosology for diagnosing and reporting vascular anomalies/malformations. This perplex have led to indiscriminate, inappropriate, and interchangeable use of terminologies while describing these vascular lesions, often resulting in incorrect diagnosis, unwarranted investigations, and improper treatment. It is often impossible to determine clinically and histopathologically whether the vascular lesion is a malformation or a neoplasm, with more than $50 \%$ of the vascular anomalies being diagnosed and termed incorrectly as hemangioma. With the help of three case reports of simple vascular malformations, each afflicting the capillaries, veins and lymphatics, we attempt to guide the clinicians in adhering to the International Society for the Study of Vascular Anomalies (ISSVA) classification. We anticipate that this case report shall be the framework that helps clinicians and pathologists to avoid misdiagnosis and misreporting of vascular malformations.
\end{abstract}

Keywords: vascular, malformation, anomaly, hemangioma, capillary, lymphatic, venous, cystic hygroma, international society for study of vascular anomalies, classification, portwine stain, ISSVA
Volume 6 Issue 2 - 2017

\author{
Cheriyanthal Sisupalan Jayapalan,' Anthony \\ George, ' Abdulla Mufeed, ${ }^{2}$ Ahammed \\ Noufal,' Meera Kunjumon Pynadath' \\ 'Department of Oral Pathology and Microbiology, Kerala \\ University of Health Sciences, India \\ ${ }^{2}$ Department of Oral Medicine and Radiology, Kerala University \\ of Health Sciences, India
}

\begin{abstract}
Correspondence: Anthony George, Department of Ora Pathology and Microbiology, MES Dental College, Kerala University of Health Sciences, Perinthalmanna, Malappuram District, 679338, Kerala, India, Email drantgeo@gmail.com
\end{abstract}

Received: October 27, 2016 | Published: January 26, 2017
Abbreviations: ISSVA, International society for the study of vascular anomalies; VMs, vascular malformations; CM, capillary malformations; LM, lymphatic malformations; VM, venous malformations

\section{Introduction}

Vascular Malformations (VMs) are developmental anomalies of the vascular plexus and should be differentiated from hemangiomas which are true neoplasms of the endothelial cells. ${ }^{1-3} \mathrm{VMs}$ occur in $1-1.5 \%$ of live-births and do not show predilection for any gender or race. ${ }^{1,2}$ Their etiopathogenesis are not properly understood, though advances made in molecular genetics have improved our insight with the discovery of several inherited mutations. ${ }^{4,5}$ The International Society for the Study of Vascular Anomalies (ISSVA) updated April 2014 classification differentiates vascular lesions with proliferative endothelium (vascular tumours) (Table 1) from structural anomalies (vascular malformations) (Table 2). ${ }^{6-9}$ An interactive portable document format of ISSVA classification is available at www.issva. org [accessed 23 April 2016]. VMs are always present at birth, though they may sometimes become clinically visible only later during early childhood. ${ }^{1-5}$ They persist throughout life and grow slowly with the person's biological growth. ${ }^{1-5}$ Unfortunately $50 \%$ of the VMs are diagnosed and termed incorrectly as hemangioma because of lack of update and knowledge of these lesion among clinicians and since it is often impossible to differentiate clinically and histopathologically a malformation from a neoplasm. ${ }^{6-9}$ The goal of this article is to serve as a guide for clinicians, pathologists and researchers on arriving at the right term while diagnosing and reporting VMs. With the help of the clinical findings of three simple VMs (capillary, lymphatic, and venous) we have attempted to discuss the different aspects of these developmental defects. We anticipate that this case series with review of literature shall ensure avoidance of out-dated, inappropriate, perplexing and interchangeable use of nosology.

Table I Vascular Tumours-modified International Society for the Study of Vascular Anomalies (ISSVA) updated April 20 I 4 classification

\begin{tabular}{cl}
\hline Benign & \\
\hline I. & Infantile hemangioma \\
i. & Focal \\
ii. & Multifocal \\
iii. & Segmental \\
iv. & Indeterminate \\
\hline
\end{tabular}


Table Continued...

\begin{aligned} & \hline Benign \\ & \hline 2. Congenital hemangioma $(\mathrm{CH}) \\ &$ i. $\quad$ Rapidly involuting $(\mathrm{RICH}) \\ &$ ii. $\quad$ Non involuting $(\mathrm{NICH}) \\ &$ iii. $\quad$ Partially involuting (PICH) \\ & 3. Spindle cell hemangioma \\ & 4. Epitheloid hemangioma \\ & 5. Pyogenic granuloma (lobular capillary hemangioma) \\ & 6. Other rare benign tumours \end{aligned}

\section{Locally Aggressive or Borderline}
a. Kaposiform hemangioendothelioma
b. Retiform hemangioendothelioma
c. Papillary intralymphatic angioendothelioma (Dabska tumor)
d. Composite hemangioendothelioma
e. Kaposi sarcoma
f. Other rare borderline tumours

\section{Malignant}
a. Angiosarcoma
b. Epitheloid hemangioendothelioma
c. Other rare malignant tumours

Table 2 Vascular Malformations (VMs)-modified International Society for the Study of Vascular Anomalies (ISSVA) updated April 2014 classification
A. Simple Malformations
I. Capillary Malformation (CM)
I. Cutaneous and/or mucosal (portwine stain)
a. Associated with bone and/or soft tissue overgrowth
b. Associated with CNS and/or ocular anomalies (Sturge-Weber syndrome)
c. Associated with arterio-venous malformations
d. Associated with microcephaly
e. Associated with megalencephaly and polymicrogyria
2. Nevus simplex (Salmon patch, angel kiss, stork bite)
3. Telangiectasia
a. Hereditary haemorrhagic telangiectasia (HHT)
b. Other rare telangiectasias

4. Cutis marmorata telangiectatica congenital (CMTC)

5. Other rare $\mathrm{CM}$

\section{Lymphatic Malformation (LM)}

I. Common (cystic)
a. Macrocystic
b. Microcystic
c. Mixed cystic

2. Generalized Lymphatic Anomaly (GLA)

3. Associated with Gorham-Stout disease 
Table Continued..

\section{Lymphatic Malformation (LM)}

4. Channel-type

5. Primary lymphedema
a. Nonne-Milroy syndrome
b. Primary hereditary lymphedema
c. Lymphedema-distichiasis
d. Hypotrichosis-lymphedema-telangiectasia
e. Primary lymphedema with myelodysplasia
f. Primary generalized lymphatic anomaly
g. Microcephaly with or without chorioretinopathy, lymphedema, mental retardation
h. Lymphedema-choanal atresia

6. Other rare LM

\section{III.Venous Malformation (VM)}

I. Common
a. Focal
b. Multifocal
c. Diffuse

2. Familial VM cutaneo-mucosal (VMCM)

3. Blue rubber bleb nevus (Bean) syndrome

4. Glomuvenous malformation (GVM)

5. Cerebral cavernous malformation (CCM)

6. Other rare VM

IV.Arterio-venous Malformation (AVM)

I. Sporadic

2. Associated with $\mathrm{HHT}$

3. Associated with CM-AVM

4. Other rare AVM

Arterio-venous Fistula (AVF) - Congenital

I. Sporadic

2. Associated with $\mathrm{HHT}$

3. Associated with CM-AVM

4. Other rare AVF

\section{Other rare simple malformations}

\section{B. Combined Malformations}
I. Capillary-VM
2. Capillary-LM
3. Capillary-AVM
4. Lymphatic-VM
5. Capillary-lymphatic-VM
6. Capillary-lymphatic-AVM
7. Capillary-venous-AVM
8. Capillary-lymphatic-AVM

\section{Malformations of major named vessels}

D. Malformations associated with other anomalies 


\section{Case presentation}

\section{Case I}

A 37 yr. old woman reported with bleeding gums while brushing her teeth since the last $3 \mathrm{wk}$. An extraoral examination revealed a reddish-pink macule involving the right ophthalmic and maxillary dermatome (Figure 1A). She reported to have had this 'birthmark' since infancy. Her peri-orbital and malar areas bilaterally had diffuse blackish pigmentation. The facial skin over the right maxilla and forehead had a rough texture. The right half of her upper lip was thick with deeper lip-lines and redder in colour in comparison to the left half and the lower lip. The enlargement was non-pulsatile and without thrill. Her right pupil was lighter brown in colour in comparison to the dark-brown pupil of the left eye. She had lost her vision in the right eye. She gave no history of seizures and was not on any medication for any systemic presentation. Intraoral examination revealed poor oral hygiene with chronic generalized gingivitis and grossly decayed right mandibular second premolar along with first and third molars. The right maxillary labial and buccal mucosa (Figure 1B), vestibule, gingiva, and hard palate from the midline were redder in colour in comparison to the other quadrants. A soft red smooth surfaced $1 \times 2 \mathrm{~cm}$ sessile swelling that bled on probing was present in the buccal marginal gingiva of right maxillary canine and first premolar. A routine orthopantomograph revealed generalized periodontal bone loss and multiple grossly decayed mandibular molars having well-defined radiolucent periapical lesions. She was advised skull radiography, angiography and contrast enhanced MRI for further diagnosis of her vascular lesion, but the patient was unwilling for such investigations due to the asymptomatic nature of the facial and lip lesion. She declined ophthalmic consultation and investigation. Routine blood examination revealed normal parameters. Her systolic and diastolic blood pressures were within the normal range of her age. The consulting physician reported the vascular lesion as portwine stain with hemangioma of upper lip. At our centre, based on the clinical history, appearance, and physical examination a provisional diagnosis of capillary malformation (portwine stain-with soft tissue overgrowth or Sturge-Weber syndrome) was arrived at by the examining pathologist. The reported chief presenting complaint of bleeding gums was treated with quadrant-wise sub-gingival oral prophylaxis and curettage followed by laser surgical excision of the gingival mass. The grossly decayed mandibular molars were surgically removed under local anaesthesia containing adrenaline, with minimal trauma to the associated tissues, and the resultant bleeding could be adequately controlled with compression packs, surgical haemostatic sponge and suturing. The excised gingival lesion on histopathological examination showed ulcerated keratinized stratified squamous surface epithelium with focal areas of pyogenic membrane. Underlying fibrovascular connective tissue had numerous budding capillaries lined by single layer of endothelial cells (some congested with RBC's), and dense chronic inflammatory cell infiltrate (predominantly lymphocytes). Histopathologically the lesion was diagnosed as pyogenic granuloma (Figure 1C). The patient is under bi-annual periodic follow-up over the last two yr. and till-date no further complication associated with the vascular malformations has been reported. She has been adequately maintaining her oral hygiene by performing correct brushing technique and the advised oral hygiene measures.

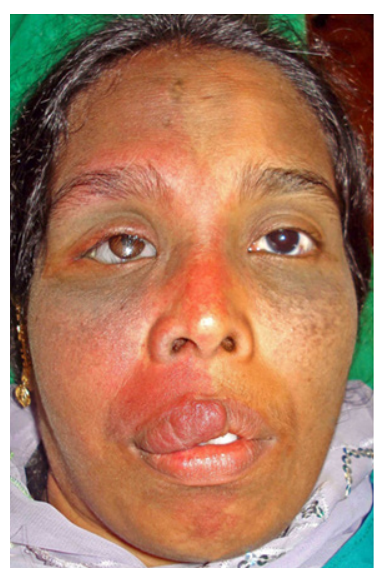

Figure IA Diffuse reddish-pink macule of the right face with prominent right half of upper lip and blindness of right eye due to capillary malformations.

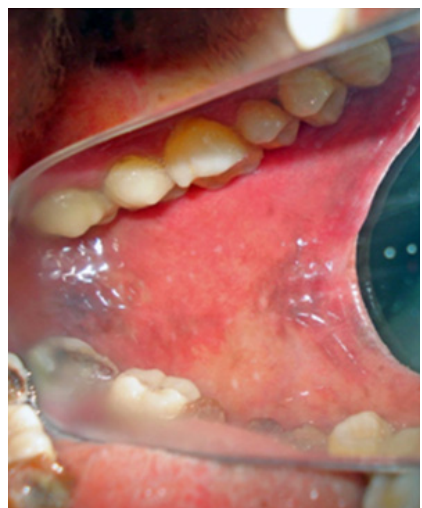

Figure IB Right upper buccal mucosa is redder in colour in comparison to the other mucosal areas.

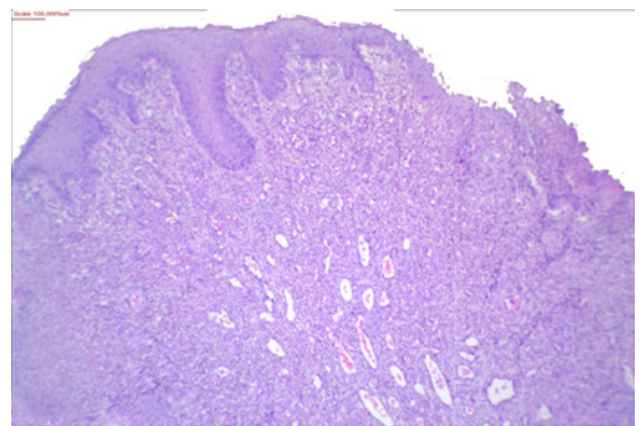

Figure IC I0x photomicrograph showing vascular arrangement of pyogenic granuloma.

\section{Case 2}

A 28 yr. old man reported with a complaint of vesicle formation on right buccal mucosa which aggravated 1 month ago after placement of fixed orthodontic appliance by a general practitioner for correction of crowding of mandibular anteriors. He gave a history of such multiple lesions being present since childhood. He gave no history of any discharge, though the vesicle ruptured discharging clear salty fluid when accidentally bitten upon. He had undergone excisional surgeries of these lesions before without any clinical improvement. He carried 
no medical records but claimed that histopathological examination after a surgical intervention was reported as lymphangioma. Extraoral examination showed a mid-facial asymmetry restricted to the right cheek and involving the upper and lower lips (Figure 2A). Intraoral examination revealed difficulty in mouth opening and multiple translucent thick-walled ovoid vesicles of $<2 \mathrm{~cm}^{3}$ that produced a pebbly surface on the right buccal mucosa extending from the commissure of mouth along the plane of occlusion to the retromolar pad (Figure 2B). On palpation the vesicles where non-tender, noncompressible and non-pulsatile. The treating clinician referred the patient with a differential diagnosis of vesiculo-bullous lesion, salivary gland tumour, and lymphangioma. The examining physician at our teaching hospital made a clinical diagnosis of microcystic lymphatic malformation of the right buccal mucosa based on the clinical history and physical examinations. The patient was advised contrast enhanced MRI for evaluation of the extent and size of the lesion. The patient refused further investigations and requested for symptomatic relief with medications and avoidance of surgical or other interventions. He was referred back to his treating clinician with instructions to evaluate the patient for lingual orthodontics or for removable aligners and retainers for achieving dental esthetics without traumatising the buccal mucosa. The patient discontinued the fixed orthodontic treatment and decided against correction of his misaligned lower anteriors. He has not reported for any further evaluation and has been lost to follow-up.

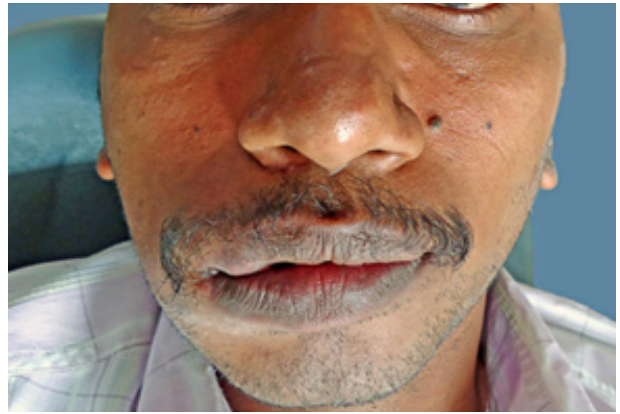

Figure 2A Mid-facial asymmetry involving right cheek due to lymphatic malformation.

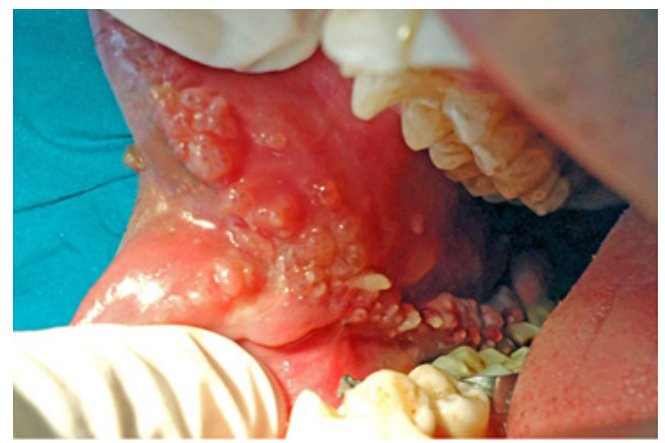

Figure 2B Pebbly translucent vesicles on right buccal mucosa

\section{Case 3}

A 21 yr. old male reported with purplish-blue soft tissue enlargement on the left side of the tongue that caused difficulty in speech, swallowing, and frequent accidental biting of the tongue. He gave a clinical history of the discolouration being present from birth and that the lesion slowly enlarged in size with his biological growth to a nodular swelling. Intraoral examination revealed left hemi-macroglossia with focal areas of prominent tongue papillae and hyperkeratosis. He had purplish-blue localized nodules on the tip, left posterior lateral and left ventral part of his tongue (Figures $3 \mathrm{~A} \& 3 \mathrm{~B}$ ). Nodular lesions were palpable in the neck at the posterior submandibular and high jugular region. The skin over the neck nodules was without discolouration and had normal texture and appearance. The intraoral and the neck nodules were soft and compressible on palpation, emptied on digital pressure, were tender, non-pulsatile and without thrill. The examining junior physician diagnosed the lesion as cavernous hemangioma with cystic hygroma. On review the senior consultants based on the clinical history and the intraoral appearance arrived at a provisional diagnosis of multifocal venous malformation afflicting the tongue and neck or a combined lymphatic-venous malformation. Due to the patient's financial constraint and lack of medical insurance he was referred to a governmental tertiary care centre for further evaluation, investigation and treatment. The patient reportedly underwent further investigations at the referral hospital and had wide surgical excision with partial glossectomy and embolization through neck dissection (the investigatory and treatment details were not shared with the authors by the referral hospital). A post three yr. evaluation when the patient reported for an unrelated ailment showed that the nodular lesions could only be identified on palpation and the patient was in good health and spirit. He did report of mild speech impairment in pronunciations of certain words, occasional stuttering, and itching sensation of the tongue. (Patient refused permission for follow-up clinical photographs).

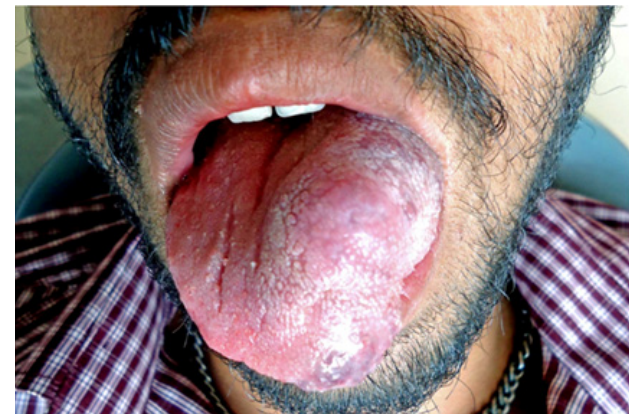

Figure 3A Hemi-macroglossia due to venous malformation.

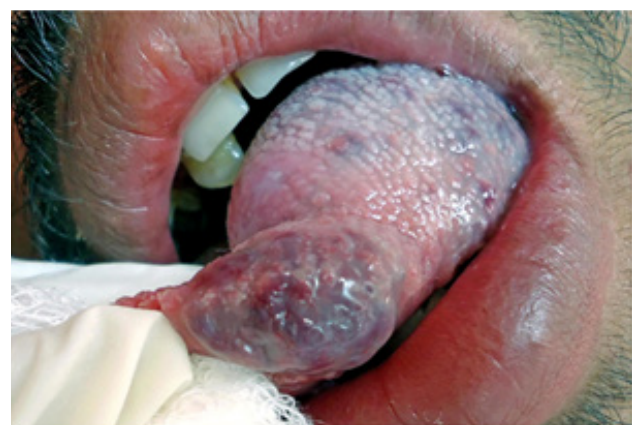

Figure 3B Localized purplish-blue nodules on anterior lateral tongue.

\section{Discussion}

The Greek suffix 'oma' means cellular tumoral proliferation and thus the term hemangioma should never be used for describing VMs which are developmental anomalies. ${ }^{1,6-11}$ Hemangiomas are usually not present at birth, arise by $8 \mathrm{wk}$. of life, exhibits a rapid proliferative phase to develop into a raised rubbery bright-red tumour mass, and is followed by a involutive phase. $90 \%$ of hemangiomas disappear by the age of 9 yr. by involution. ${ }^{7-13}$ Growth and expansion of VMs unlike hemangioma is by distension and hypertrophy (rather than 
hyperplasia), and usually do not show a growth phase. The anomaly may enlarge in response to trauma, changes in intravascular pressure, infections, or hormonal changes during pregnancy and puberty. ${ }^{1,2}$ VMs are uncommon and occur in $1-1.5 \%$ of births, but the actual incidence of VMs may probably be more, since many clinicians erroneously report them as hemangioma, which is the most commonly used imprecise term for VMs. ${ }^{1-3}$

\section{Capillary (Venular) Malformations (CM) can be grouped into}

a. Cutaneous and/or mucosal CM (portwine stain, telangiectatic nevus, nevus flammeus).

b. Nevus simplex (stork bite, angels kiss, Salmon stain/patch).

\section{c. Teleangiectasia. ${ }^{1,4,7}$}

Cutaneous and mucosal CM commonly called portwine stain occur in $0.4-1 \%$ of new-born and appear as reddish-pink macules without any thrill or bruit over facial dermatomes supplied by the branches of the trigeminal nerve. ${ }^{1-15}$ They occur because of distension of post-capillary venules due to defect in the sensory and autonomic vascular innervations of the capillary plexus, commonly resulting from the somatic mutation of GNAQ and RASA-1 gene. ${ }^{1-11}$ $90 \%$ of the portwine stains affect more than one dermatomes, with the combined affliction of maxillary and ophthalmic dermatomes being the most common. ${ }^{1-10}$ With age the lesions darken in colour and thicken to produce a violaceous coloured cobblestone surfaced vascular lesion. ${ }^{1-11}$ The afflicted area usually shows hypertrophy of the associated jaw bones, gingiva, oral mucosa, and lips, leading to interdental spacing, malocclusion, and unaesthetic thick sagging lip. ${ }^{1-11} 10 \%$ of portwine stains are associated with Sturge-Weber syndrome which is characterized by $\mathrm{CM}$ mainly involving the maxillary and ophthalmic dermatomes, hemifacial hyperplasia, and ipsilateral leptomeningeal venous malformations of the cerebral cortex that is diagnosed by the presence of gyriform "tramline" calcification on skull images. ${ }^{1-15}$ They are usually associated with ophthalmic manifestations, convulsive disorder, mental retardation, and contra lateral hemiplegia. ${ }^{1-15}$ Nevus simplex affects the midline of the head, forehead, eyelids, and/or glabella, as a red patch/stain., ${ }^{1,7}$ They usually lighten and disappear before 5 yr. of age. ${ }^{1,7}$ Hereditary haemorrhagic telangiectasia (HTT) is an inherited autosomal dominant mucocutaneous disorder affecting 1 in 5-8000 and is associated with mutations commonly in ENG, ACVRL-1, and SMAD-4 gene. ${ }^{12,13}$ The Curacao criteria helps in arriving at a definite diagnosis of HHT if at least three of the following clinical manifestations are present

a. Spontaneous recurrent epistaxis.

b. Multiple mucocutaneous telangiectasia involving fingertip, lips, oral mucosa, or tongue,

c. Gastrointestinal, pulmonary, hepatic, cerebral, or spinal arteriovenous malformations,

\section{d. A first-degree relative with these clinical presentations. ${ }^{4-16}$}

Based on the clinical history, appearance, and physical examinations our case 1 was provisional diagnosed as capillary malformation (portwine stain associated with soft tissue overgrowth), though the treating physician had erroneously reported it as portwine stain with hemangioma of upper lip. The bleeding gingival growth was provisionally diagnosed as pyogenic granuloma (lobular capillary hemangioma). Our patient had ophthalmic manifestation (blindness) but no histories of seizures, mental retardation or paraplegia. We were unable to rule out Sturge-Weber syndrome as the patient was unwilling to be evaluated for leptomeningeal venous malformations.

Incidence of Lymphatic Malformations (LM) is 1 in 2-4000 births, occurring due to obstruction or sequestration of primitive lymphatic vessels with resultant failure of drainage from these areas. ${ }^{9-19} 75$ $90 \%$ of LM occurs in the neck, followed by axilla, mediastinum, and retroperitoneum. ${ }^{1-19} \mathrm{LM}$ are categorized depending on the depth and size of the lesion into

a. Microcystic (out-dated terms: capillary lymphangioma, superficial lymphangioma, lymphangioma circumscriptum, lymphangioma simplex)

b. Macrocystic (out-dated terms: lymphangioma cavernosum, cystic hygroma, lymphangioma cysticum).

c. Mixed (combined micro and Macrocystic LM) ${ }^{1-20}$ Within the oral cavity microcystic LM has been reported commonly on the anterior $2 / 3$ of the tongue, palate, gingiva, and mucosa. ${ }^{1,18}$

They appear as multiple translucent non-compressible vesicles of $<2 \mathrm{~cm}^{3}$ containing viscous clear fluid producing a pebbly or warty surface resembling 'frog spawn' or 'tapioca pudding'. ${ }^{1-15}$ Macrocystic LM appear as localized painless non-pulsatile multiple cysts of $>2 \mathrm{~cm}^{3}$ commonly found in the supra-clavicular fossa of the posterior triangle and in the cervical area just below the angle of the mandible. ${ }^{1-19}$ They have a rubbery compressible consistency, are not warm to touch, produce no bruit or thrill, and are covered by normal appearing skin unless haemorrhage or communication with venous malformation produce a blue discolouration. ${ }^{1-19}$ Gorham-Stout disease (vanishing bone disease) is characterized by LM of single or multiple bones and neighbouring soft tissues, with progressive osteolysis and often pathological fractures. ${ }^{7}$ The terms 'lymphangioma' and 'cystic hygroma' have been discarded. ${ }^{6-11}$ Our case 2 was inappropriately differentially diagnosed as lymphangioma by the referral clinician, and was later at our centre it was appropriately reported as microcystic lymphatic malformation. The multiple microcystic vesicles on the buccal mucosa were thick walled, had clear fluid, and were less than $2 \mathrm{~cm}^{3}$ in size.

Venous Malformations (VM) has a reported incidence of 1 in 10,000 with the common VM being the most commonly seen variant. This has been categorized into unifocal, multifocal and diffuses..$^{7-17}$ $93 \%$ of VM are unifocal, with their most common site of occurrence being the head, neck, and limbs. ${ }^{4-17}$ The tongue, palate, lips, and jaw bones are the most common areas afflicted in the maxillofacial region. ${ }^{1}$ They clinically appear as soft compressive nodular lesions that usually empty on application of finger pressure, produce no thrill or bruit, are not warm to touch, and their colour varies from purple for superficial lesions to blue or normal mucosal colour for the deep lesions. ${ }^{1-17}$ Multifocal VM appear as multiple small $<5 \mathrm{~cm}$ asymptomatic raised pink to dark blue superficial nodules that are present from birth. ${ }^{7,17}$ The nodules produce pain on compression, usually involve only the superficial mucosa and rarely invade the underlying deeper tissues..$^{7-17}$ Thrombosis of the malformed veins occur frequently due to the sluggish flow of blood in these vessels, resulting in pain on palpation and observation of organized thrombi or calcifications (phleboliths) on imaging. ${ }^{7-17}$ Blue rubber bleb (Bean) syndrome presents as small round, dark hyperkeratotic bleb-like or nipple-like multiple lesions on the skin of the soles and palms. ${ }^{7}$ They are associated with anemia due to chronic bleeding from VM of gastrointestinal tract. ${ }^{7}$ Glomuvenous malformations (out-dated terms: glomangioma, glomangiomatosis) manifest as dark blue or purple coloured non-compressible nodular or plaque-like lesions of the skin, that are often painful to palpation 
and have a cobblestone clinical appearance. ${ }^{7}$ Combined vascular malformations are simple malformations, malformations of major named vessels, or a combination of both types, and present themselves as two or more malformations of vasculature in a single lesion. ${ }^{7}$ Our case 3 was initially inappropriately diagnosed as cavernous hemangioma with cystic hygroma, but after re-consideration of the clinical history, appearance and physical findings a provisional diagnosis of multifocal venous malformation of the tongue and neck or a combined lymphatic-venous malformation was made. The patient had multifocal dark blue to violaceous coloured nodular lesions that were less than $5 \mathrm{~cm}$ in diameter. Since the patient underwent detailed investigation and treatment at the government tertiary centre, to which the authors could not get access, a confirmatory diagnosis of combined lymphatic-venous malformation could not be arrived.

Diagnosis of vascular anomalies can be made by clinical history and physical findings, with a minority requiring imaging studies and even smaller number requiring biopsy and histopathological examination. ${ }^{9,21}$ Doppler-ultrasound, magnetic resonance imaging, computerized topography, and angiography are necessary to identify the size, location, extent, and collateral feeders before any therapeutic or surgical interventions are attempted. ${ }^{9,21}$ The management of VMs depends on the type and flow characteristics of the malformation, the functional impairment, disfigurement, the threat to life, and on the expertise of the surgeon or the interventional radiologist. ${ }^{9,21}$ The therapeutic modalities include sclerotherapy, embolization, laser photocoagulation, and surgical excision and are beyond the scope of this article. ${ }^{9,21}$

\section{Conclusion}

International Society for the Study of Vascular Anomalies (ISSVA) has constantly strived to provide consistent, accurate and updated terminologies for vascular lesions with the help of the current understanding of the genetic causes of these lesions. Lack of update, knowledge and understanding of the vascular lesions by the healthcare personals has led to inappropriate, perplexing and interchangeable use of terminology while diagnosing VMs. It is anticipated that this case report shall provide a framework and serve as a guide for pathologists, clinicians, and researchers in diagnosing VMs. We hope that this article shall encourage clinicians in using the correct Nosology and avoid labelling all vascular lesions as hemangioma.

\section{Consent}

Written informed consent was obtained from all the three patients for publishing and sharing the medical and investigatory records along with any images for academic advancement and knowledge.

\section{Acknowledgments}

None.

\section{Funding}

None.

\section{Conflicts of interest}

The authors declare there is no conflict of interests.

\section{References}

1. Redondo P. Vascular Malformations (1) Concept, Classification, Pathogenesis and Clinical Features. Actas Dermosifiliogr. 2007;98(3):141-158
2. Martines F, Imordino V. Arteriovenous malformation of the base of tongue in pregnancy: case report. Acta Otorhinolaryngol Ital. 2009;29(5):274 278.

3. Mulliken JB, Glowacki J. Hemangiomas and vascular malformations in infants and children: a classification based on endothelial characteristics. Plast Reconstr Surg. 1982;69(3):412-422.

4. Boon LM, Ballieux F, Vikkula M. Pathogenesis of Vascular Anomalies. Clin Plast Surg. 2011;38(1):7-19.

5. Legiehn GM, Heran MK. A Step-by-Step Practical Approach to Imaging Diagnosis and Interventional Radiologic Therapy in Vascular Malformations. Semin Intervent Radiol. 2010;27(2):209-231.

6. Enjolras O, Wassef M, Chapot R. Color atlas of vascular tumours and vascular malformations. 1st ed. New York, USA: Cambridge University Press; 2007.

7. Wassef M, Blei F, Adams D, et al. Vascular anomalies classification: recommendations from the international society for the study of vascular anomalies. Pediatrics. 2015;136(1):e203-e214.

8. George A, Mani V, Noufal A. Update on the classification of hemangioma. J Oral Maxillofac Pathol. 2014;18(Suppl 1):117-120.

9. George A, Jayapalan CS, Noufal A. Diagnosing hemangioma and vascular malformations of head and neck. Health Sci. 2014;3(1):JS002C.

10. Jayapalan CS, George A, Pynadath MK, et al. Venular (Capillary) Vascular Malformation of Maxillofacial Region: Portwine Stain. Ora Maxillofac Pathol J. 2014;5(2):491-493.

11. Richter GT, Friedman AB. Hemangiomas and Vascular Malformations: Current Theory and Management. Int J Pediatr. 2012;2012:645678.

12. Donnelly LF, Adams DM, Bisset GS. Vascular malformations and hemangiomas: a practical approach in a multidisciplinary clinic. $A m J$ Roentgenol. 2000;174(3):597-608.

13. Restrepo R, Palani R, Cervantes L, et al. Hemangiomas revisited: the useful, the unusual and the new. Pediatr Radiol. 2011;41(7):895-915.

14. Dadras SS, North PE, Bertoncini J, et al. Infantile hemangiomas are arrested in an early developmental vascular differentiation state. Mod Pathol. 2004;17(9):1068-1079.

15. Neville BW, Damm DD, Allen CM, et al. Soft tissue tumours. Oral and maxillofacial pathology. 3rd ed. Missouri, USA: Saunders, St Louis; 2009:507-552.

16. Govani FS, Shovlin CL. Hereditary haemorrhagic telangiectasia: a clinical and scientific review. Eur J Hum Genet. 2009;17(7):860-871.

17. Dompmartin A, Vikkula M, Boon ML. Venous Malformation: update on etiopathogenesis, diagnosis \& management. Phlebology. 2010;25(5):224 235.

18. Stanescu L, Georgescu EF, Simionescu C, et al. Lymphangioma of the oral cavity. Rom J Morphol Embryol. 2006;47(4):373-377.

19. Mirza B, Ijaz L, Saleem M, et al. Cystic hygroma: an overview. J Cutan Aesthet Surg. 2010;3:139-144.

20. Khunger N. Lymphatic malformations: current status. J Cutan Aesthet Surg. 2010;3(3):137-138.

21. Cooke D, Ghodke B, Natarajan SK, et al. Embolization in the Head and Neck. Semin Intervent Radiol. 2008;25(3):293-309. 